\title{
Detection of Sugars via Chirality Induced in Europium (III) Compounds
}

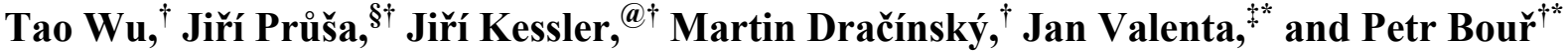

†Institute of Organic Chemistry and Biochemistry, Academy of Sciences, Flemingovo náměstí 2, Prague 6, 16610, Czech Republic,

${ }^{\S}$ Department of Analytical Chemistry University of Chemistry and Technology, Prague, Technická 5, Prague 6, 166 28, Czech Republic.

${ }^{\circledR}$ Department of Physical and Macromolecular Chemistry, Faculty of Science, Charles University, Hlavova 8, 12840 Prague, Czech Republic

$\$$ Faculty of Mathematics and Physics, Charles University, Ke Karlovu 3, 12116, Prague 2, Czech Republic

Corresponding Authors: bour@uochb.cas.cz,jan.valenta@mff.cuni.cz

\section{Contents}

Figure S1. Raman and ROA spectra of $\mathrm{EuCl}_{3}, \mathrm{NaEuEDTA}$ and $\mathrm{Na}_{2} \mathrm{EuDEPA}$ solutions in the presence of the four monosaccharides.

Figure S2. Simulated Raman/luminescence and ROA/CPL spectra of a $\mathrm{Eu}^{3+}$ complexes with EDTA, DEPA, $\alpha$ - and $\beta$-mannose.

Figure S3. Luminescence excitation and emission spectrum for EuDEPA.

Figure S4. Comparison of parts of $\mathrm{EuCl}_{3}$, EuEDTA and EuDEPA luminescence spectra.

Figure S5. Example of luminescence decay changes for $698 \mathrm{~nm}$ band of EuEDTA.

Figure S6. Free energy profiles. 

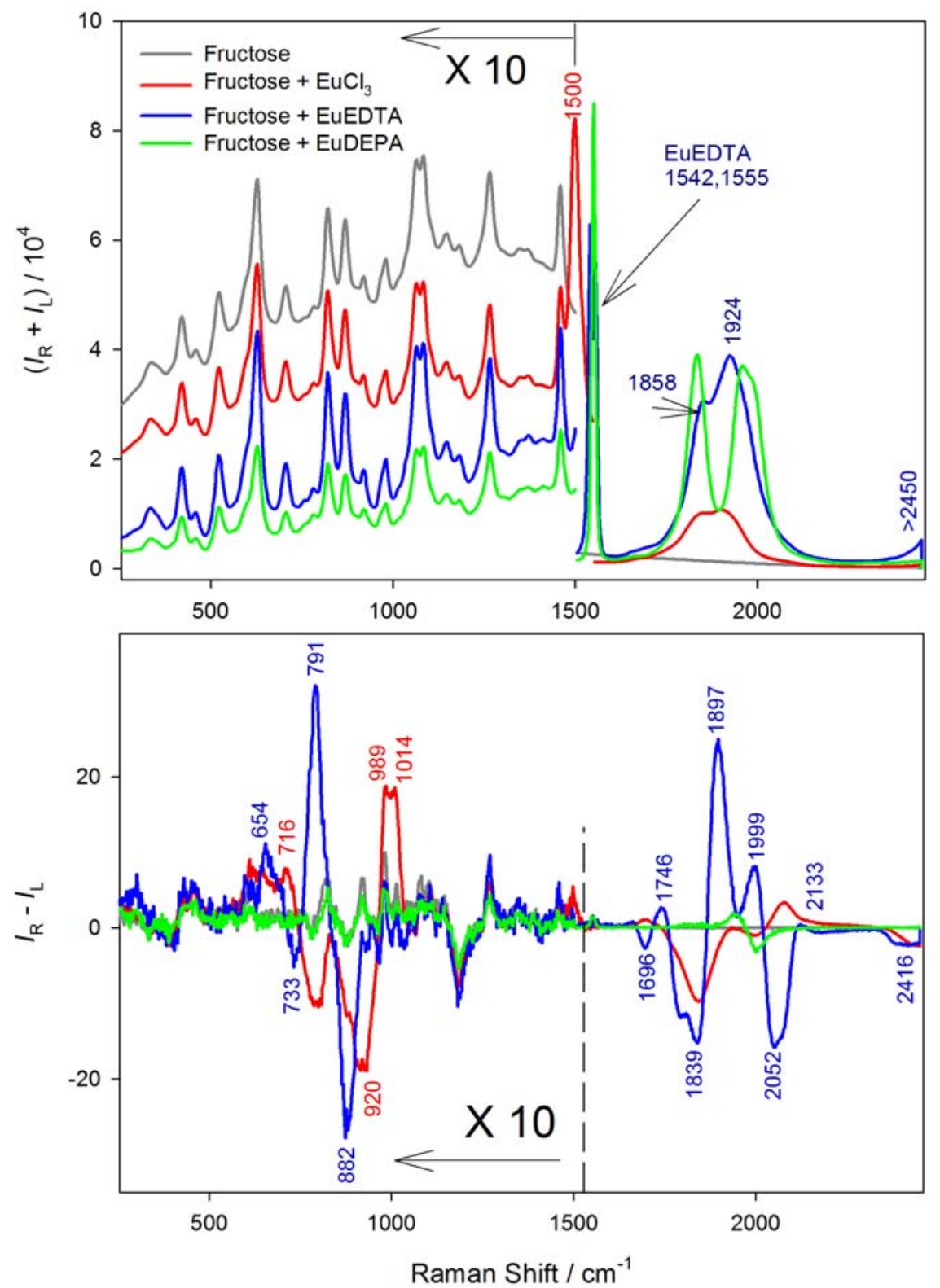

Figure S1 (this and next three pages). Raman and ROA spectra of $\mathrm{EuCl}_{3}, \mathrm{Na}_{2} \mathrm{EuEDTA}$ and $\mathrm{Na}$ EuDEPA solutions in the presence of the four monosaccharides. 

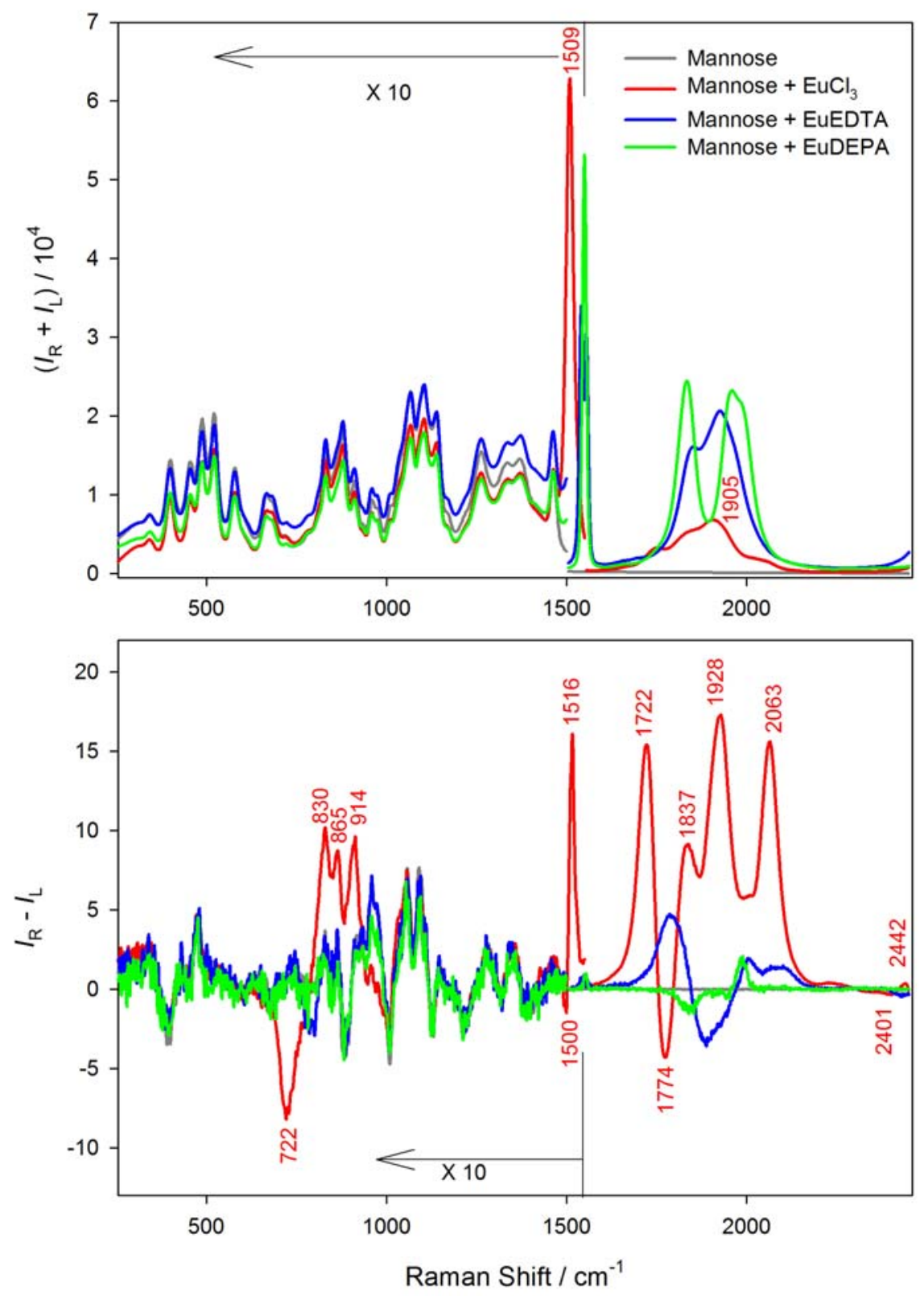

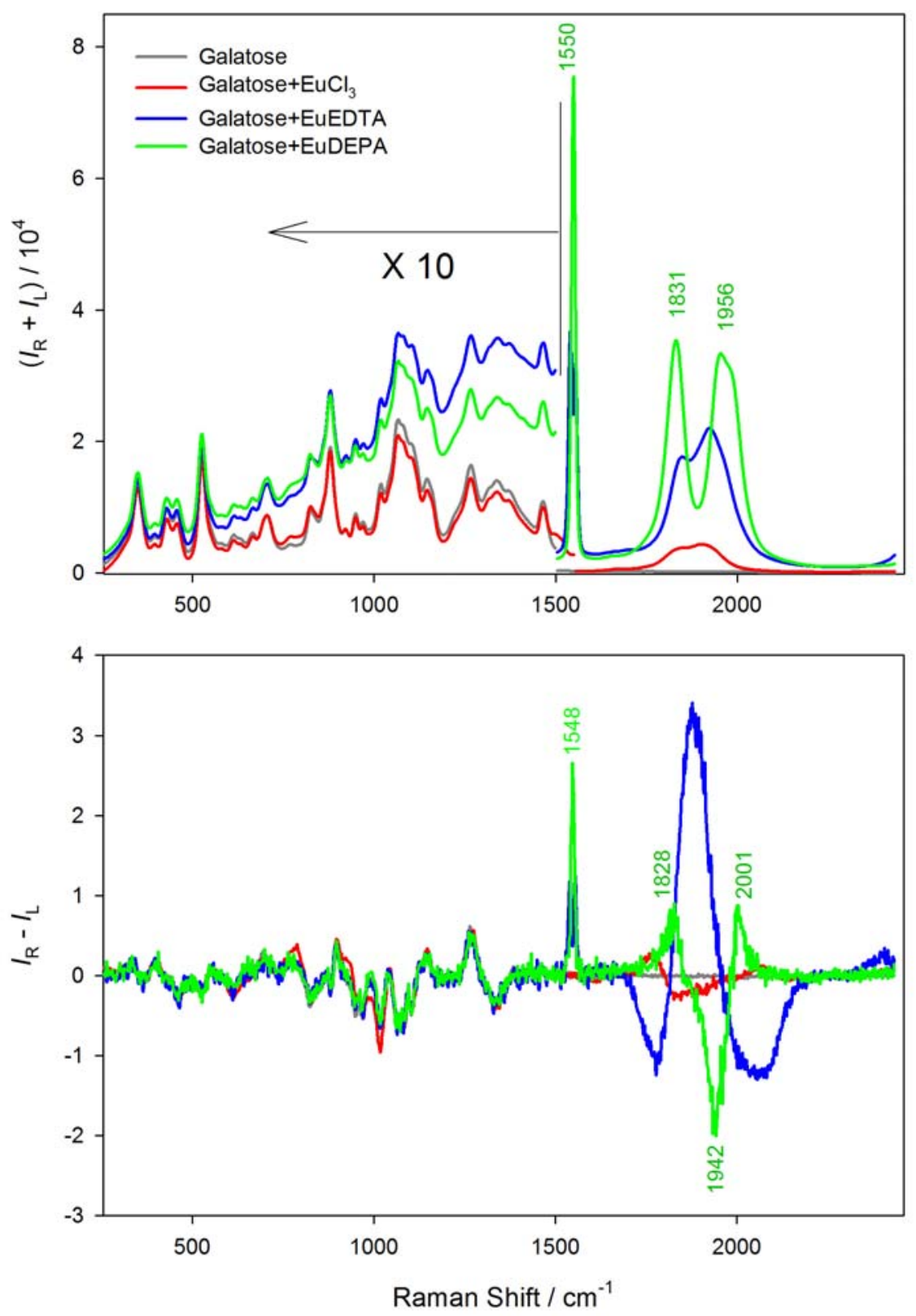

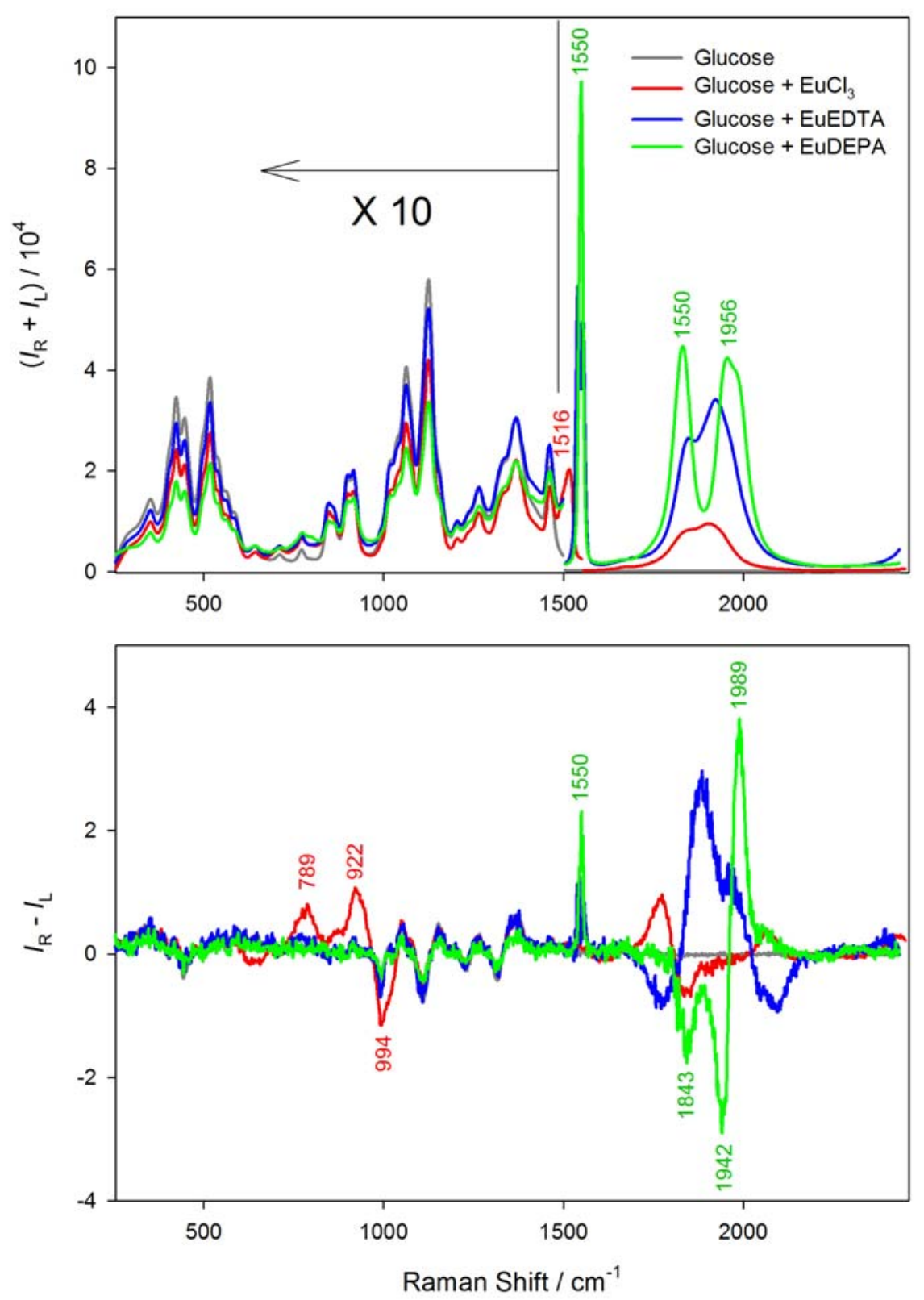

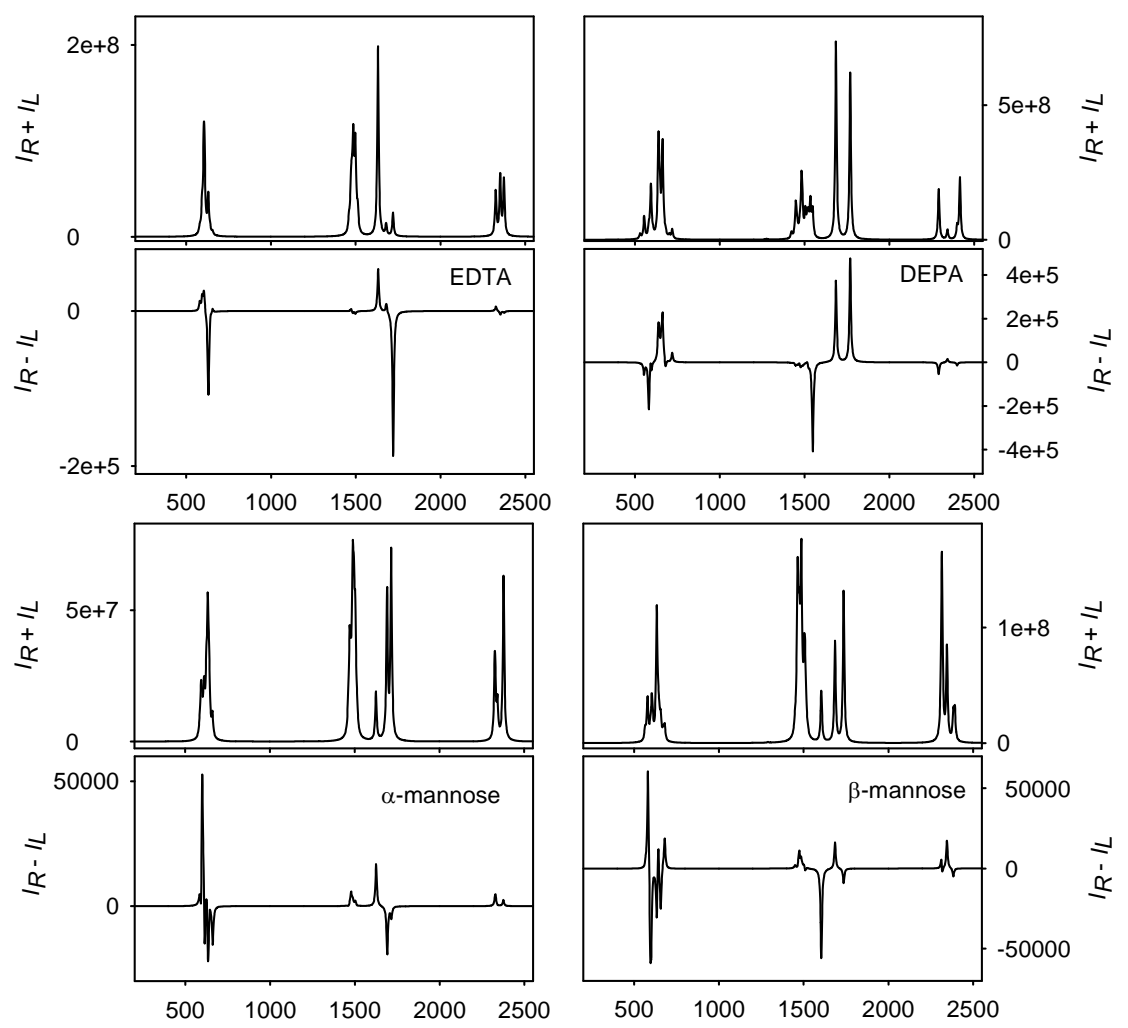

Wavenumber $/ \mathrm{cm}^{-1}$

Figure S2. Simulated Raman/luminescence and ROA/CPL spectra of a $\mathrm{Eu}^{3+}$ complexes with EDTA, DEPA, $\alpha$ - and $\beta$-mannose. 


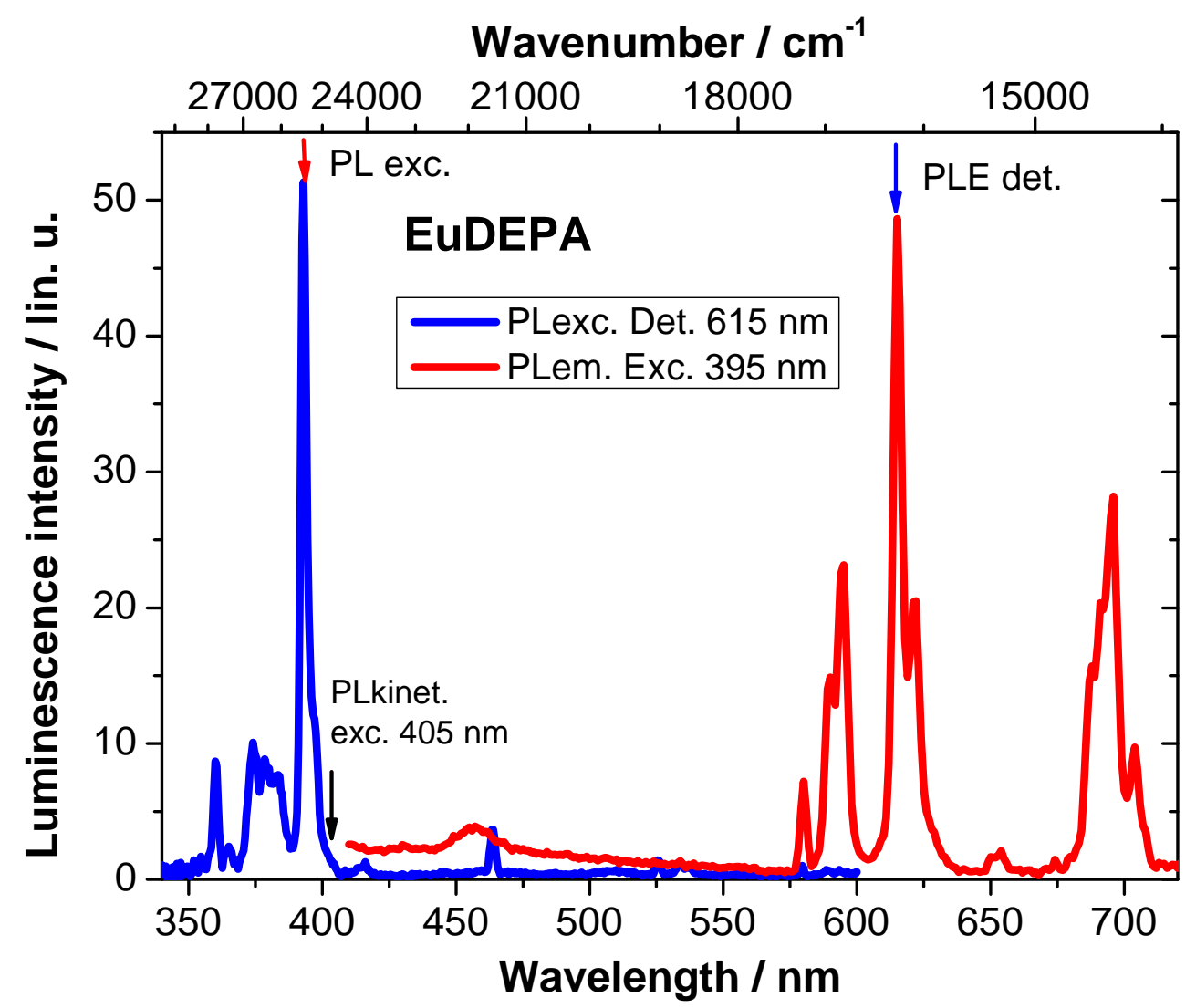

Figure S3. Luminescence excitation and emission spectrum for EuDEPA. Excitation spectrum (blue line) is detected at $615 \mathrm{~nm}$, while emission spectrum (red line) is excited at $395 \mathrm{~nm}$. 


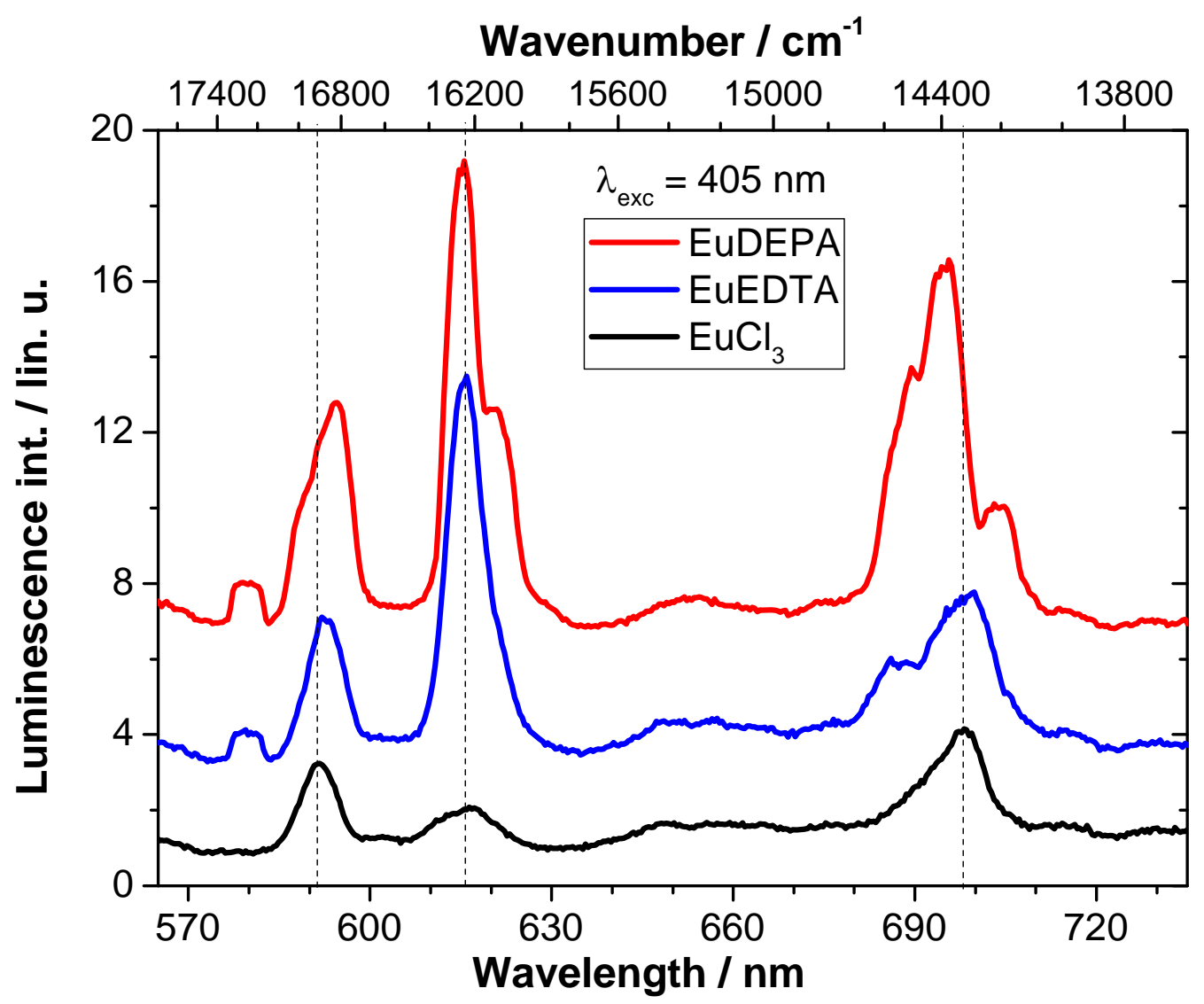

Figure S4. Comparison of parts of $\mathrm{EuCl}_{3}$, EuEDTA and EuDEPA luminescence spectra, demonstrating shifts and splitting of emission lines. 


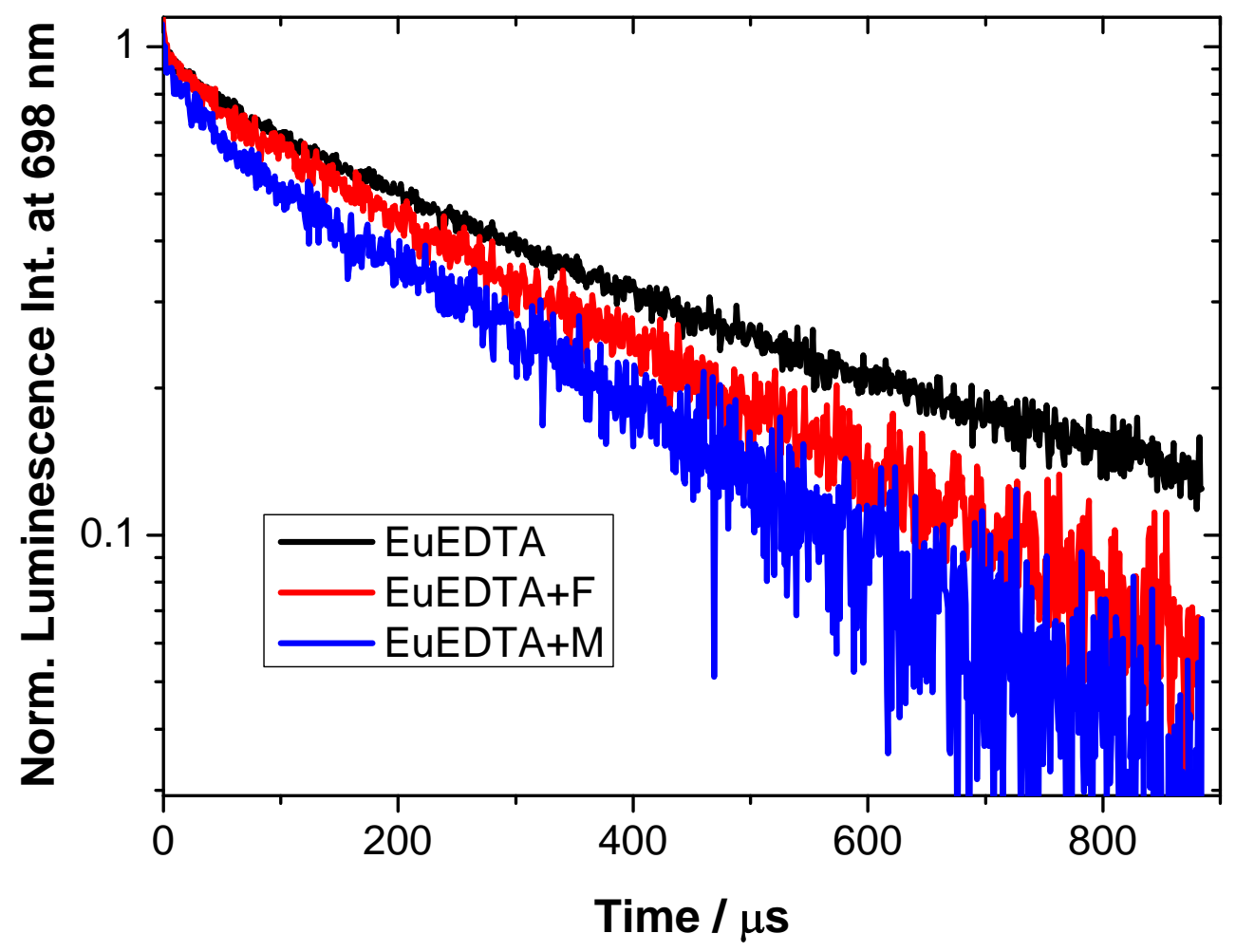

Figure S5. Example of luminescence decay changes for $698 \mathrm{~nm}$ band of EuEDTA induced by addition of fructose or mannose. The addition of the sugars causes shortening of the decay times. 


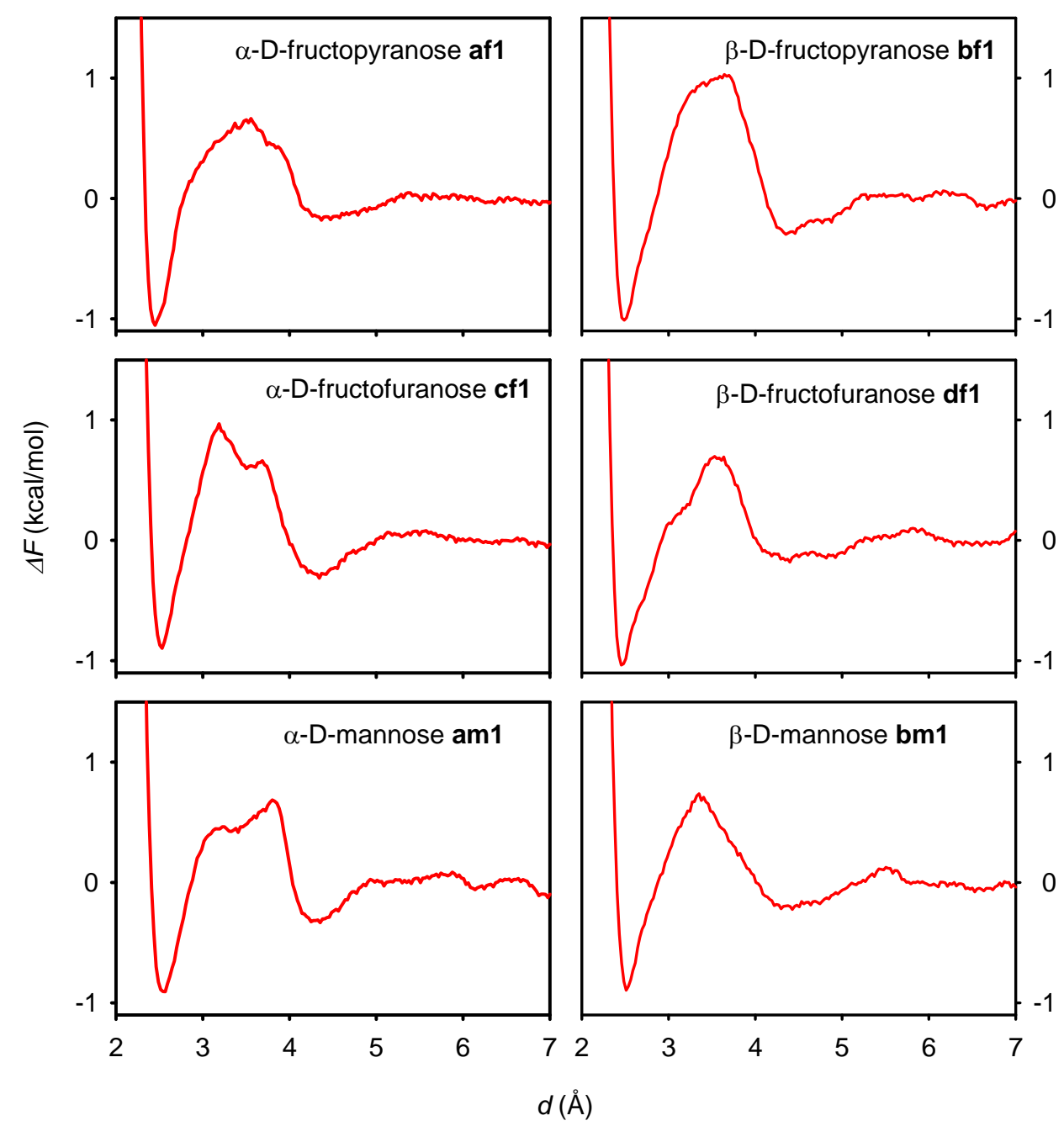

Figure S6. Free energy profiles obtained by WHAM for different fructose and mannose forms. 